\title{
Dynamical Formation of Kerr Black Holes with Synchronized Hair: An Analytic Model
}

\author{
Carlos A. R. Herdeiro and Eugen Radu \\ Departamento de Física da Universidade de Aveiro and CIDMA, Campus de Santiago, 3810-183 Aveiro, Portugal
}

(Received 28 June 2017; revised manuscript received 2 October 2017; published 28 December 2017)

\begin{abstract}
East and Pretorius have successfully evolved, using fully nonlinear numerical simulations, the superradiant instability of the Kerr black hole $(\mathrm{BH})$ triggered by a massive, complex vector field. Evolutions terminate in stationary states of a vector field condensate synchronized with a rotating $\mathrm{BH}$ horizon. We show that these end points are fundamental states of Kerr BHs with synchronized Proca hair. Motivated by the "experimental data" from these simulations, we suggest a universal (i.e., field-spin independent), analytic model for the subset of BHs with synchronized hair that possess a quasi-Kerr horizon, applicable in the weak hair regime. Comparing this model with fully nonlinear numerical solutions of BHs with a synchronized scalar or Proca hair, we show that the model is accurate for hairy BHs that may emerge dynamically from superradiance, whose domain we identify.
\end{abstract}

DOI: 10.1103/PhysRevLett.119.261101

Introduction.-A 50 year-old lingering question in black hole $(\mathrm{BH})$ physics has been the end point of the Kerr BH superradiant instability [1,2], triggered by massive, bosonic fields [3-10]. Apart from its theoretical interest, this instability offers an unexpected opportunity for testing the existence of ultralight bosonic particles suggested by beyond the standard model scenarios, e.g., [11]. BHs effectively become particle detectors [12], creating a remarkable synergy between strong gravity, particle physics, and astrophysics, testable by ongoing or future gravitational waves and electromagnetic observations (see, e.g., [12-28]).

In a recent breakthrough, East and Pretorius [1] reported long-term numerical evolutions of this instability, using a Proca field to trigger it. Their evolutions lead to equilibrium states wherein the $\mathrm{BH}$ horizon angular velocity, $\Omega_{H}$, synchronizes with the phase angular velocity of the (complex) Proca field. This suggests that these endpoints belong to the family of Kerr BHs with synchronized Proca hair (KBHSPH), previously constructed as fully nonlinear, stationary solutions of the Einstein-Proca system [29].

In the present Letter, we establish that the equilibrium states obtained dynamically in [1] correspond to fundamental states of $\mathrm{KBHSPH}$, making these $\mathrm{BHs}$ the first dynamical counter example to the no-hair conjecture [30], in general relativity, with a simple, physically reasonable matter content. Moreover, we identify which of these hairy BHs can be end points of superradiance.

More generically, for a subset of BHs with synchronized bosonic hair, including all solutions that may dynamically form from the superradiant instability, we propose an analytic model based on the hypothesis that the horizon is quasi-Kerr. This holds when the BH hair is weak, but it also even holds for considerably hairy BHs when the matter field is dilute. Within this model, explicit, universal (i.e., field-spin independent) formulas for physical quantities are presented, in terms of a "hairiness" parameter. These provide an analytic handle for further studies of these solutions in this interesting regime.

The quasi-Kerr horizon (QKH) model.-Consider a stationary, axisymmetric, asymptotically flat, $\mathrm{BH}$ with synchronized hair of a generic bosonic matter field $\psi$, with field mass $\mu$. Synchronization means that $\Omega_{H}=w / m$ where $\psi \sim e^{-i(w t-m \phi)} \ldots, \partial / \partial t, \partial / \partial \phi$ are the Killing vector fields associated to stationarity and axisymmetry, respectively, and $m \in \mathbb{Z}^{+}$and $w$ is the field's frequency. Known examples have been constructed for both scalar [31-34] and vector matter [29] (see, e.g., Sec. II in [32] for details). The Arnowitt-Deser-Misner (ADM) mass and angular momentum are $M$ and $J$, while the corresponding horizon data are $M_{H}, J_{H}$ (computed as Komar integrals) together with the area, $A_{H}$, and temperature, $T_{H}$ and $\Omega_{H}$. The corresponding matter field data obey (see e.g., [29])

$$
M_{(\psi)}=M-M_{H}, \quad J_{(\psi)}=J-J_{H} .
$$

The following Smarr relation also holds that $M=$ $\frac{1}{2} T_{H} A_{H}+2 \Omega_{H}\left[J-J_{(\psi)}\right]+M_{(\psi)}$, or, equivalently

$$
M_{H}=\frac{1}{2} T_{H} A_{H}+2 \Omega_{H} J_{H} .
$$

Furthermore, the solutions satisfy the first law of thermodynamics $d M=\frac{1}{4} T_{H} d A_{H}+\Omega_{H} d J$.

It is convenient to define the aforementioned physical quantities normalized by the ADM mass,

$$
\begin{aligned}
j & \equiv \frac{J}{M^{2}}, \quad a_{H} \equiv \frac{A_{H}}{16 \pi M^{2}}, \quad w_{H} \equiv \Omega_{H} M, \\
t_{H} & \equiv 8 \pi M T_{H},
\end{aligned}
$$

and to introduce two measures of the "hairiness," which provide, respectively, the fraction of energy (angular momentum) in the matter field 


$$
p \equiv \frac{M_{(\psi)}}{M}, \quad q \equiv \frac{J_{(\psi)}}{J} .
$$

In the absence of hair $(p=0=q)$, i.e., for Kerr,

$j=\frac{4 w_{H}}{1+4 w_{H}^{2}}, \quad a_{H}=\frac{1}{1+4 w_{H}^{2}}, \quad t_{H}=1-4 w_{H}^{2}$,

with $0 \leq w_{H} \leq 1 / 2$, as the limits corresponding with the Schwarzschild solution and extremal Kerr, respectively.

In the presence of hair $(p \neq 0 \neq q)$, we assume that the $\mathrm{BH}$ horizon is quasi-Kerr. We expect this to hold in the weak hair regime, $p, q \ll 1$ [35]. Under this assumption, we introduce the quasi-Kerr horizon (QKH) model approximating the horizon quantities by those of a Kerr BH, but with the replacements $(M, J) \rightarrow\left(M_{H}, J_{H}\right)$ :

$$
\begin{gathered}
\Omega_{H}=\frac{M_{H}}{2 J_{H}}(1-\chi), \\
A_{H}=8 \pi M_{H}^{2}(1+\chi), \quad T_{H}=\frac{\chi}{4 \pi M_{H}(1+\chi)},
\end{gathered}
$$

where $\chi \equiv \sqrt{1-J_{H}^{2} / M_{H}^{4}}$. For the Kerr family, these formulas are exact, but for hairy BHs, they are just an approximation.

For hairy BHs, (2) still holds; from (1), together with the first law of thermodynamics and the QKH model, we find that the matter field mass and angular momentum obey

$$
d M_{(\psi)}=\Omega_{H} d J_{(\psi)} .
$$

We formally integrate (8), treating $\Omega_{H}$ as an input parameter. This is justified regarding the hairy $\mathrm{BH}$ as a composed system of a horizon plus a matter distribution. Then $\Omega_{H}$ characterizes the horizon subsystem, which is external (albeit coupled) to the "hair" subsystem. Then

$$
M_{(\psi)}=\Omega_{H} J_{(\psi)}, \quad \text { i.e., } \quad M-M_{H}=\Omega_{H}\left(J-J_{H}\right) .
$$

An equivalent form of (9) reads

$$
p=w_{H} j q
$$

Relations (6) and (10) give two constraints for the four variables $\left(p, q, w_{H}, j\right)$. We may choose $p, w_{H}$ as the independent (control) parameters yielding the simple expressions

$q=p \frac{1+4(1-p)^{2} w_{H}^{2}}{p+4(1-p)^{2} w_{H}^{2}}, \quad j=\frac{p+4(1-p)^{2} w_{H}^{2}}{w_{H}\left(1+4(1-p)^{2} w_{H}^{2}\right)}$,

which are, thus, predictions of the QKH model that shall be compared against the fully nonlinear (numerical) solutions in the next section. Similarly,

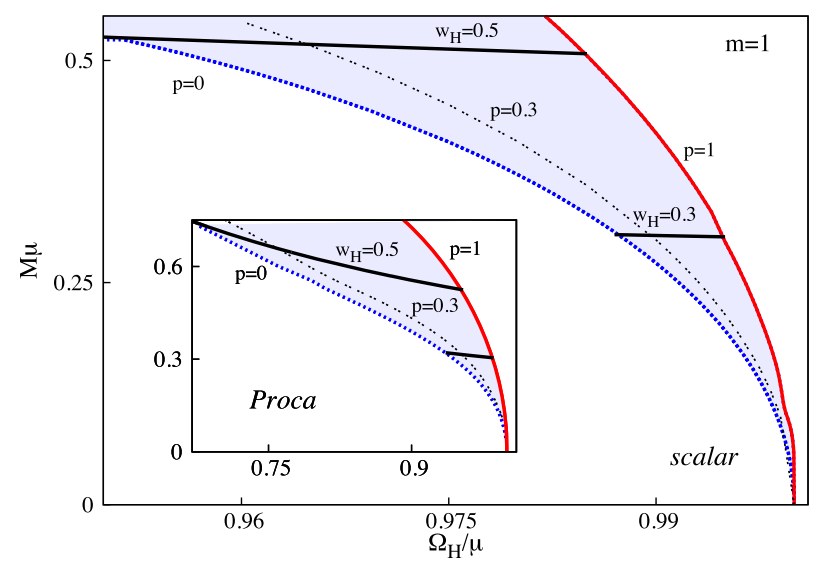

FIG. 1. Part of the domain of existence of Kerr BHs with scalar (main panel) or Proca (inset) hair (shaded blue region) in a $M$ vs $\Omega_{H}$ diagram, both in units of $\mu$. The Kerr limit-existence line ( $p=0$, blue-dotted line) — and the solitonic limit ( $p=1$, redsolid line) are shown, together with the line $p=0.3$ and the lines $\omega_{H}=0.3 ; 0.5$, in both panels.

$a_{H}=\frac{(1-p)^{2}}{1+4(1-p)^{2} w_{H}^{2}}, \quad t_{H}=\frac{1-4(1-p)^{2} w_{H}^{2}}{1-p}$.

Observe the Kerr relations (5) are recovered as $p=0$.

QKH model vs numerical solutions.-To test the $\mathrm{QKH}$ model, we compare it with fully nonlinear (numerical) BHs with synchronized hair, which are solutions of the corresponding Einstein-matter system. To test universality, we shall consider both the scalar solutions constructed in $[31,32]$ and the Proca solutions. The latter are nodeless solutions, but otherwise, they are within the family introduced in [29], cf. the Supplemental Material (SM) [40]. The corresponding part of the domain of existence to be explored is shown in Fig. 1 [46].

Figure 1 shows the neighborhood of the existence line, wherein the hairy BHs reduce to vacuum $\operatorname{Kerr}(p=0)$. We shall be particularly interested in the hairy BHs that may arise from the growth of the superradiant instability of Kerr. Then, thermodynamics imposes an upper bound of $p \leq 1-1 / \sqrt{2} \simeq 0.29$. The corresponding BHs exist in the allowed strip between the $p=0$ and $p=0.3$ lines in Fig. 1. The complete domain of existence can be found in [31] (Fig. 5 below) for the scalar (Proca) case.

In Fig. 2, we test the QKH model by comparing the model's results for $\mathcal{Y}=\left\{j, a_{H}, t_{H}\right\}$ with the data from the numerical solutions. It shows the relative errors $\left|1-\mathcal{Y}^{(\text {th })} / \mathcal{Y}^{(\text {num })}\right|$ for the allowed strip with $p \in[0,0.3]$, $\omega_{H} \in[0,0.5]$. This analysis shows that for the solutions with $j<1$, whose importance will be detailed below, the relative errors are below the percent level for all physical quantities, and typically lower for the scalar case. The errors are still only at a few percent level when $p \sim 0.3$ and for low values of $\omega_{H}$, the relative errors are below $1 \%$, even 

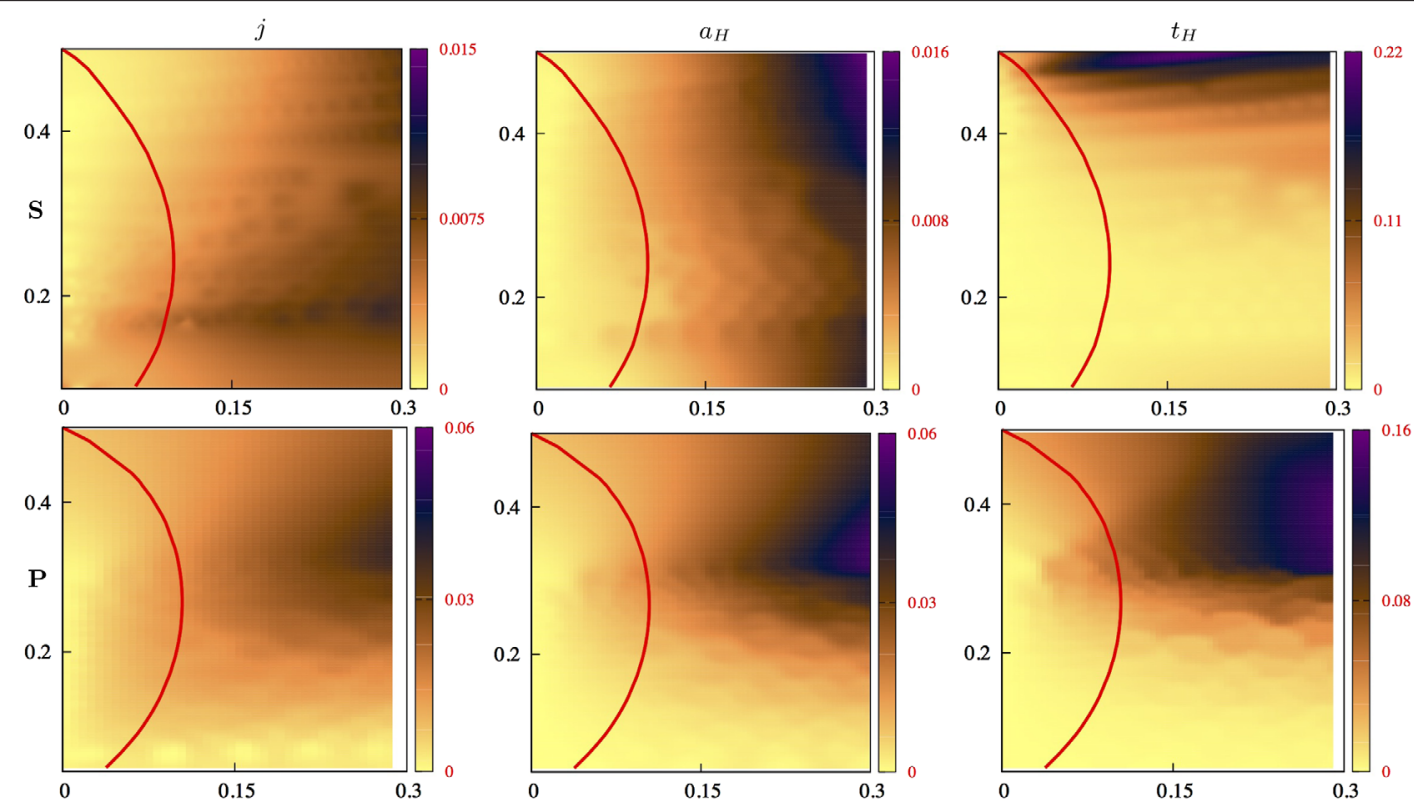

FIG. 2. The relative errors are shown in the allowed strip on the ( $\left.p, w_{H}\right)$-plane for the reduced angular momentum $j$ (left panels), area $a_{H}$ (middle panels), and temperature $t_{H}$ (right panels) for both BHs with scalar (S-top panels) and Proca (P-bottom panels) hair. The red lines are the set of solutions with $j=1$; solutions to the left of these lines have $j<1$.

for $p \sim 0.3$. We interpret this unexpected accuracy in the description as a consequence of a separation of scales. Indeed, $\omega_{H}<\mu M$, and $M$ (the gravitational radius of the $\mathrm{BH}$ ), and $\mu$ (the inverse Compton wavelength of the massive bosonic field) are the two fundamental scales of the problem. When the dimensionless coupling $M \mu$ is small—often called the Newtonian regime - the bosonic field is diluted, even though it may correspond to a large fraction of the total spacetime energy [47]. In this regime, it is expected that the BH horizon is not strongly affected by the (mostly far away) bosonic field distribution, being quasi-Kerr. This type of separation of scales is often used to alleviate the complexity of physical problems. Two examples in the context of strong gravity appear in the study of spinning $\mathrm{BH}$ binaries [48] and the blackfold approach to higher dimensional BHs [49].

The analysis of Fig. 2 validates the QKH model for the region where $j<1$, but we emphasize that even for $j>1$, the model can be accurate, especially for small $\omega_{H}$. This is illustrated in Fig. 3, where we exhibit a $a_{H}$ vs $j$ plot. Even for $j$ approaching 2 (thus for non-Kerr $\mathrm{BHs}$ ) the analytic model fits well the numerical points, along the lines of constant $\omega_{H}$, for both the scalar and Proca case, supporting its (matter field spin) universality.

Dynamical formation from superradiance.-The QKH model was suggested by a phenomenological observation in [1]. In Fig. 3 therein, a Kerr-like approximation was observed to accurately fit the equilibrium state obtained from the numerical simulations of the growth of the superradiant instability, triggered by a Proca field. To establish that such equilibrium states are the hairy BHs with a quasi-Kerr horizon that we have described before, Fig. 4 below mimics Fig. 3 in [1], wherein the normalized irreducible mass, $M_{H}$ and $J_{H}$ are plotted. In the formalism introduced above, these quantities translate as $M_{\text {ir }} / M=\sqrt{a_{H}}, \quad M_{H} / M=1-p$ and $J_{H} / M^{2}=j(1-q)$.

Figure 4 shows that the QKH model accurately fits our solutions of both Kerr BHs with scalar or Proca hair, as it did fit the equilibrium BHs obtained through numerical evolutions by East and Pretorius in [1]. This establishes that the equilibrium states obtained through the growth of the superradiant instability are the fundamental states of Proca BHs with synchronized hair, cf. the SM, and in particular, the ones with a quasi-Kerr horizon. It also predicts a similar result for the scalar case: superradiance forms BHs with synchronized scalar hair and a quasi-Kerr horizon (see [19] for related observations).

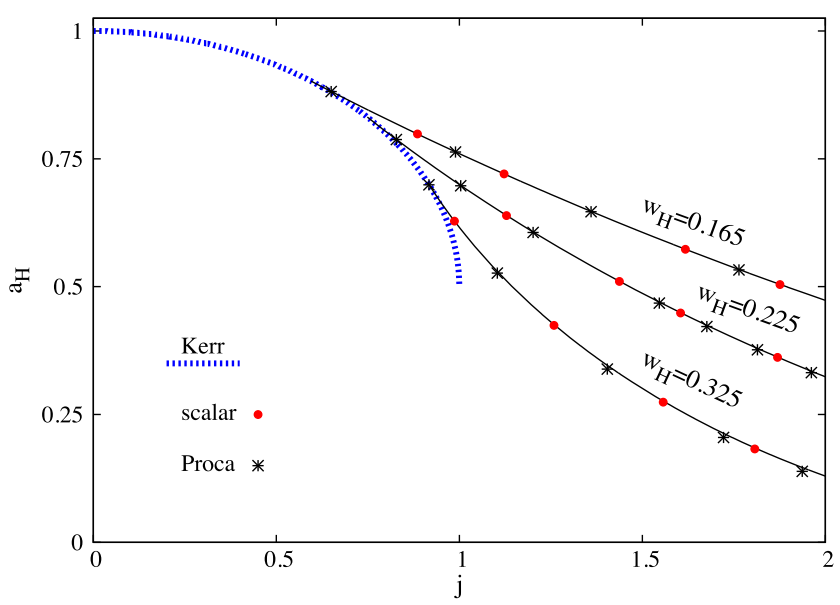

FIG. 3. $j$ vs $a_{H}$ diagram. The black stars are Proca numerical solutions; the red points are for scalar hairy BHs. The curves correspond to the analytical model for fixed $\omega_{H}$ values. 


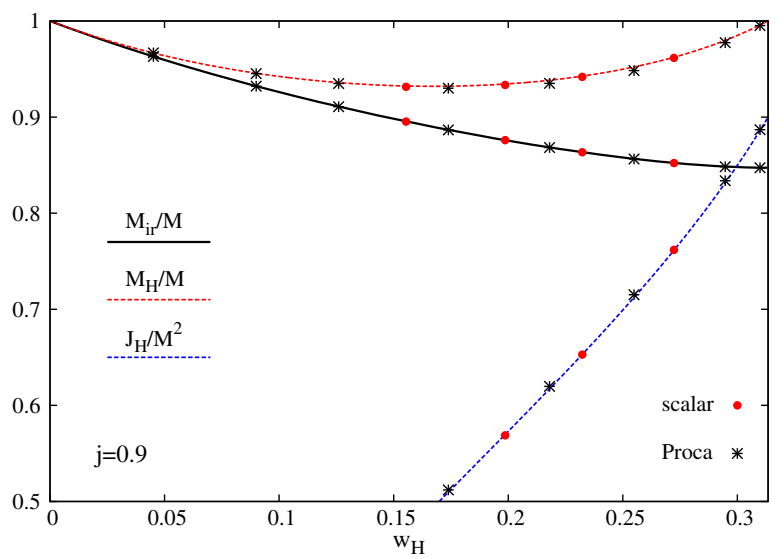

FIG. 4. Normalized irreducible mass, horizon mass, and angular momentum: numerical solutions (points) vs the analytic model (curves). Here we have taken $j=0.9$ (in [1] the authors took $j=0.99$ ) to show the universality of the agreement.

We can now investigate how the dynamics of superradiance migrates a vacuum Kerr $\mathrm{BH}$ into a $\mathrm{BH}$ with synchronized hair. This is exhibited in Fig. 5, for the examples of the numerical evolutions in [1]. Under the assumption used therein, that a single superradiant mode is present (the fastest growing mode), which implies that axisymmetric is a good approximation during the evolution, radiation is negligible [1], and the total mass and angular momentum are preserved. The process is, thus, conservative. This implies that the migration in Fig. 5-an ADM mass vs horizon angular velocity plot-occurs along a horizontal line, ending at a hairy $\mathrm{BH}$ with the same $j$ as the initial Kerr solution. We have verified there is a unique such solution-cf. the inset in Fig. 5. In particular, this implies the equilibrium $\mathrm{BH}$ with synchronized hair has $j \leq 1$. Solutions with $j \leq 1$ exist in a substrip of the allowed strip $p<0.3$. This explains our emphasis in this region in Fig. 2. Using Eq. (11), one shows, moreover, that $j \leq 1 \Rightarrow p \lesssim 0.0973$, a stronger bound on the hairiness that can form dynamically from superradiance.

Hairy BHs are entropically favored.-The (vacuum Kerr) $\rightarrow$ (hairy $\mathrm{BH}$ ) migration is conservative (in the above description), but it is irreversible. Thermodynamics determines the arrow of time. To understand this, we resort to the QKH model, which allows us to show that the hairy BH is always entropically favored (as observed in [31] from the numerical data). Indeed, from the $a_{H}(j, p)$-cubic equation: $a_{H}^{3}-2(1-p) a_{H}^{2}+\left[j^{2} / 4+(1-p)^{2}\right] a_{H}=j^{2}(1-p)^{2} / 4$, the solution for small $p$ [up to $\left.\mathcal{O}\left(p^{3}\right)\right]$ reads

$a_{H}=a_{H}^{(\text {Kerr })}+\left(\frac{\left(1+\sqrt{1-j^{2}}-\frac{1}{2} j^{2}\right)\left(1+\sqrt{1-j^{2}}\right)}{j^{2} \sqrt{1-j^{2}}}\right) p^{2}$.

Thus, for the same $M, J$, the hairy $\mathrm{BH}$ is entropically favored over the Kerr BH. In other words, the direction of

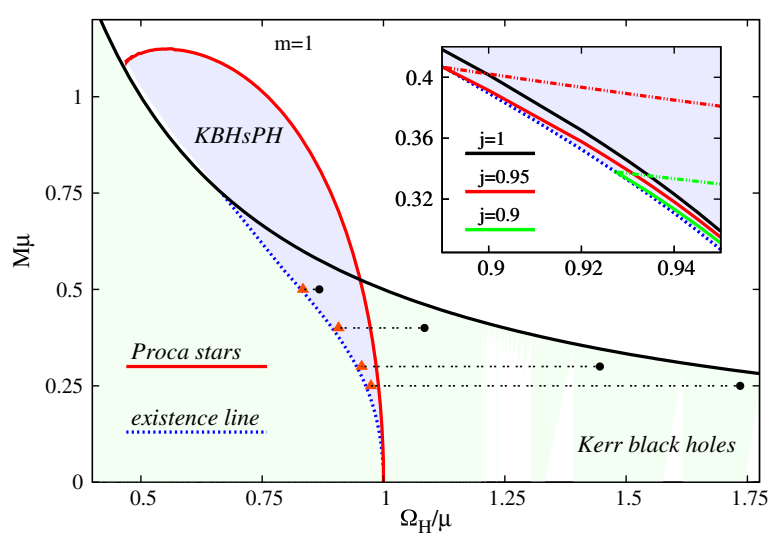

FIG. 5. (Main panel) Domain of existence of fundamental states of KBHSPH (shaded-blue region). Vacuum Kerr BHs exist below the black-solid line (corresponding to extremal Kerr). The horizontal-dotted lines show the migration trajectories from vacuum Kerr BHs (black dots) to hairy BHs (red triangles) of the evolutions in [1]. The inset shows constant $j$ lines for both vacuum Kerr (dashed) and hairy BHs (solid), which always meet at the existence line, for any $j<1$. Migration of a Kerr BH with spin $j$ terminates when the horizontal line (constant $M$ ) meets a hairy $\mathrm{BH}$ with that $j$ value. See [9] for an earlier discussion of adiabatic evolutions, in the scalar case, based on linear theory.

the migration in Fig. 5 is determined by the second law of thermodynamics.

Remarks.-It was observed in [31] that there is a nonuniqueness for Kerr BHs with synchronized scalar hair (as there is in the Proca case). Near the Kerr limit, this degeneracy is discrete and of degree two: fixing $M, J$, there exists a hairy $\mathrm{BH}$ and a vacuum Kerr BH. The numerical simulations reported in [1], together with the QKH analytic model we have proposed and the fundamental states of KBHSPH described in the SM, support the conclusion that these degenerate states correspond to the initial (Kerr) and final (hairy) states of the superradiant instability [50]. We remark that all numerical data presented herein are from stationary solutions we have generated, and the comparison with the data in [1] is performed only via the QKH model. It would be interesting to perform a direct comparison with the latter numerical data, at the level of the geometry and matter distribution.

When more than one mode (and with different values of $m$ ) becomes important during the superradiant evolution, the axisymmetry assumption in [1] may not be accurate and less smooth evolutions may occur, cf. the results reported in nonasymptotically flat setups [51-53]. In this case, BHs with synchronized hair and a quasi-Kerr horizon may be transient equilibrium states. One may imagine, for instance, that the equilibrium states dynamically attained in [1], with $\omega_{\text {eq }} / m=\Omega_{H}^{\mathrm{eq}}$, may be driven to evolve by a lower frequency modes, $\omega_{\text {new }} / m<\Omega_{H}^{\mathrm{eq}}$. An evolution of $\Omega_{H}^{\mathrm{eq}}$ towards synchronizing it with $\omega_{\text {new }}$ is, however, hampered by the existing $\omega_{\text {eq }}$ mode. Understanding this process is a relevant open issue [54]. 
Finally, it would be interesting to adapt the QKH model for higher dimensional BHs with synchronized hair [56,57], including in anti-de Sitter space [58]. Another possible application of this model is in a study of the thermodynamics of BHs with synchronized hair.

We are very grateful to W. East and F. Pretorius for correspondence and V. Cardoso, P. Cunha and P. Pani for comments. C.H. and E.R. acknowledge funding from the FCT-IF programme. This work was partially supported by the H2020-MSCA-RISE-2015 Grant No. StronGrHEP-690904, the H2020-MSCA-RISE-2017 Grant No. FunFiCO-777740 and by the CIDMA Project No. UID/MAT/04106/2013. The authors would also like to acknowledge networking support by the COST Action GWverse CA16104. Computations were performed at the Blafis cluster, in Aveiro University.

[1] W. E. East and F. Pretorius, Phys. Rev. Lett. 119, 041101 (2017).

[2] W. H. Press and S. A. Teukolsky, Nature (London) 238, 211 (1972).

[3] T. Damour, N. Deruelle, and R. Ruffini, Lett. Nuovo Cimento 15, 257 (1976).

[4] T. Zouros and D. Eardley, Ann. Phys. (N.Y.) 118, 139 (1979).

[5] S. L. Detweiler, Phys. Rev. D 22, 2323 (1980).

[6] H. Furuhashi and Y. Nambu, Prog. Theor. Phys. 112, 983 (2004).

[7] S. R. Dolan, Phys. Rev. D 76, 084001 (2007).

[8] J. G. Rosa, J. High Energy Phys. 06 (2010) 015.

[9] S. R. Dolan, Phys. Rev. D 87, 124026 (2013).

[10] R. Brito, V. Cardoso, and P. Pani, Lect. Notes Phys. 906, 1 (2015).

[11] A. Arvanitaki, S. Dimopoulos, S. Dubovsky, N. Kaloper, and J. March-Russell, Phys. Rev. D 81, 123530 (2010).

[12] A. Arvanitaki and S. Dubovsky, Phys. Rev. D 83, 044026 (2011).

[13] P. Pani, V. Cardoso, L. Gualtieri, E. Berti, and A. Ishibashi, Phys. Rev. Lett. 109, 131102 (2012).

[14] H. Witek, V. Cardoso, A. Ishibashi, and U. Sperhake, Phys. Rev. D 87, 043513 (2013).

[15] H. Yoshino and H. Kodama, Prog. Theor. Phys. 128, 153 (2012).

[16] H. Yoshino and H. Kodama, Prog. Theor. Exp. Phys. (2014) $043 \mathrm{E} 02$.

[17] P. Pani and A. Loeb, Phys. Rev. D 88, 041301 (2013).

[18] A. Arvanitaki, M. Baryakhtar, and X. Huang, Phys. Rev. D 91, 084011 (2015).

[19] R. Brito, V. Cardoso, and P. Pani, Classical Quantum Gravity 32, 134001 (2015).

[20] P. V. P. Cunha, C. A. R. Herdeiro, E. Radu, and H. F. Runarsson, Phys. Rev. Lett. 115, 211102 (2015).

[21] H. Yoshino and H. Kodama, Classical Quantum Gravity 32, 214001 (2015).

[22] F. H. Vincent, E. Gourgoulhon, C. Herdeiro, and E. Radu, Phys. Rev. D 94, 084045 (2016).

[23] A. Arvanitaki, M. Baryakhtar, S. Dimopoulos, S. Dubovsky, and R. Lasenby, Phys. Rev. D 95, 043001 (2017).
[24] Y. Ni, M. Zhou, A. Cardenas-Avendano, C. Bambi, C. A. R. Herdeiro, and E. Radu, J. Cosmol. Astropart. Phys. 07 (2016) 049.

[25] M. Baryakhtar, R. Lasenby, and M. Teo, arXiv:1704.05081.

[26] M. Zhou, C. Bambi, C. A. R. Herdeiro, and E. Radu, Phys. Rev. D 95, 104035 (2017).

[27] N. Franchini, P. Pani, A. Maselli, L. Gualtieri, C. A. R. Herdeiro, E. Radu, and V. Ferrari, arXiv:1612.00038.

[28] R. Fujita and V. Cardoso, Phys. Rev. D 95, 044016 (2017).

[29] C. Herdeiro, E. Radu, and H. Runarsson, Classical Quantum Gravity 33, 154001 (2016).

[30] R. Ruffini and J. A. Wheeler, Phys. Today 24, No. 1, 30 (1971).

[31] C. A. R. Herdeiro and E. Radu, Phys. Rev. Lett. 112, 221101 (2014).

[32] C. Herdeiro and E. Radu, Classical Quantum Gravity 32, 144001 (2015).

[33] B. Kleihaus, J. Kunz, and S. Yazadjiev, Phys. Lett. B 744, 406 (2015).

[34] C. A. R. Herdeiro, E. Radu, and H. Runarsson, Phys. Rev. D 92, 084059 (2015).

[35] When $p, q \rightarrow 0$, the hair becomes a stationary cloud on the Kerr background. For the scalar case these clouds were first discussed in [36] - see also [31,37-39].

[36] S. Hod, Phys. Rev. D 86, 104026 (2012).

[37] S. Hod, Eur. Phys. J. C 73, 2378 (2013).

[38] S. Hod, Classical Quantum Gravity 32, 134002 (2015).

[39] S. Hod, J. High Energy Phys. 01 (2017) 030.

[40] See Supplemental Material at http://link.aps.org/ supplemental/10.1103/PhysRevLett.119.261101 for a discussion of the fundamental states of Kerr BHs with Proca hair, which includes Refs. [41-45].

[41] R. Brito, V. Cardoso, C. A. R. Herdeiro, and E. Radu, Phys. Lett. B 752, 291 (2016).

[42] F. Schunck and E. Mielke, Classical Quantum Gravity 20, R301 (2003).

[43] S. L. Liebling and C. Palenzuela, Living Rev. Relativity 15, 6 (2012).

[44] J. Balakrishna, E. Seidel, and W.-M. Suen, Phys. Rev. D 58, 104004 (1998).

[45] N. Sanchis-Gual, C. Herdeiro, E. Radu, J. C. Degollado, and J. A. Font, Phys. Rev. D 95, 104028 (2017).

[46] The numerical results herein are for $m=1$ being obtained from several thousands of solution points for each type of hairy BHs, with typical error estimates $<10^{-3}$.

[47] Such property, already observed in [19], is not found, as far as we know, in other models of hairy BH solutions.

[48] D. Gerosa, M. Kesden, U. Sperhake, E. Berti, and R. O’Shaughnessy, Phys. Rev. D 92, 064016 (2015).

[49] R. Emparan, T. Harmark, V. Niarchos, and N. A. Obers, Phys. Rev. Lett. 102, 191301 (2009).

[50] The stability of these final (hairy) configurations against gravitational radiation dissipation is due to the complex nature of the scalar or Proca field, and the consequent existence of a global U(1) symmetry and particle number conservation law. This is not the case for one (or many) real fields, in which case the hairy $\mathrm{BH}$ decays by emission of gravitational waves.

[51] N. Sanchis-Gual, J. C. Degollado, P. J. Montero, J. A. Font, and C. Herdeiro, Phys. Rev. Lett. 116, 141101 (2016). 
[52] P. Bosch, S. R. Green, and L. Lehner, Phys. Rev. Lett. 116, 141102 (2016).

[53] N. Sanchis-Gual, J. C. Degollado, C. Herdeiro, J. A. Font, and P. J. Montero, Phys. Rev. D 94, 044061 (2016).

[54] This process is dissipative and likely more dissipative in the Proca case [55].
[55] W. E. East, Phys. Rev. D 96, 024004 (2017).

[56] Y. Brihaye, C. Herdeiro, and E. Radu, Phys. Lett. B 739, 1 (2014).

[57] C. Herdeiro, J. Kunz, E. Radu, and B. Subagyo, Phys. Lett. B 748, 30 (2015).

[58] O. J. Dias, G. T. Horowitz, and J. E. Santos, J. High Energy Phys. 07 (2011) 115. 